\title{
AUTHOR INDEX VOLUME 17
}

\section{A}

Abi-Dargham A, 162

Adler CM, 141

Akil $\mathrm{H}, 353$

Altemus M, 100

Amador XF, 316

Andreasen NC, 230

Arborelius $\mathrm{L}_{2} 44$

Austin MC, 325

Azmitia EC, 117

\section{B}

Backström T, 370

Baldessarini RJ, 186

Baldwin RM, 162

Baltazar C, 307

Bastani B, 1

Bell K, 402

Berman N, 110

Bolos A, 18

Bowden C, 110

Bowers MB, 162

Brébion $G, 316$

Breier A, 141

Brown AS, 316

Bunney BG, 402

Bunney WE, 402

C

Camp DM, 130

Cappiello A, 246

Casper R, 110

Charney DS, 162

Chergui K, 151

Clifton A, 141

Coccaro E, 264

Cohen RM, 391

Cooper T, 316

D

Dannals RF, 175

De Wied D, 284

DeJonghe DK, 130

Detera-Wadleigh S, 100

deVegvar M, 264

D'Souza CD, 162

\section{E}

Ehlers CL, 34

Elman I, 141

Emslie G, 241

Ernst M, 391

F

Fallon J, 402

Ferré S, 82
Fibiger HC, 197

Fingado CL, 162

Fornal CA, 72

Frankhuijzen-Sierevogel AC, 284

Frazer A, 110

Fuxe K, 82

G

Gallager DW, 307

Gardner JP, 72

Geraci M, 360

Gewirtz G, 316

Goekoop JG, 284

Goldman D, 18

Gorman JM, 316

Grillner P, 151

Gründer G, 175

\section{H}

Hardy KK, 391

Harkavy-Friedman J, 316

Hashim A, 202

Heninger GR, 246, 341

Hertel P, 44

Hichwa RD, 230

Hidaka K, 27

Howard DR, 175

Huang Z, 57

Hulihan-Giblin B, 18

\section{I}

Innis RB, 162

Ino A, 274, 279

Ishigaki T, 274

J

Jacobs BL, 72

Jatlow P, 162

Javitt DC, 202

Jayathilake K, 1

Jentsch ID, 92

Jons PH, 391

Jope RS, 12

\section{K}

Kanes SJ, 162

Katz MM, 110

Keator D, 402

Kegeles LS, 293

Kinon BJ, 205

Kirby D, 34

Klein DF, 113

Klein E, 402

Kocsis J, 110

Koshiya K, 27

Koslow SH, 110
Kramer HK, 117

Kula NS, 186

Kurokawa K, 27

L

Lajitha A, 202

Lam RW, 258, 382

Laruelle M, 162

Le D, 92

Leong $\mathrm{Y}-\mathrm{M}, 100$

Levant $B, 67$

Lewis DA, 325

Liberzon I, 353

Liebenauer LL, 391

Lieberman JA, 205

Lopez A, 34

\section{M}

Maes M, 1

Malaspina D, 316

Malhotra AK, 141

Malison RT, 246, 341

Mann JJ, 293

Matochik J, 391

McCance-Katz EF, 341

McCann UD, 360

McCarthy DB, 57

McDougle CJ, 246, 341

McGrath PI, 113

Meltzer $\mathrm{H}, 1$

Miller DD, 230

Mitropoulou V, 264

Morgan CM, 360

Murphy DL, 100, 360

$\mathbf{N}$

Nagai M, 279

Najafi A, 402

Nakamura Y, 274

Nakhai B, 18

Narita M, 27

Neumeyer JL, 186

Nielsen DA, 18

Nisell M, 151

Nomikos GG, 44, 151, 197

Nyberg S, 370

O

Offord SJ, 175

Ögren SO, 82

Ohara K, 274, 279

Ohmori J, 27

O'Leary DS, 230

Owen KR, 341

NEUROPSYCHOPHARMACOLOGY 1996-VOL. 17, NO. 6 
$\mathbf{P}$

Pelton GH, 246

Petitto JM, 57

Pickar D, 141

Pinals DA, 141

Poblete JC, 117

Ponto LLB, 230

Post DL, 360

Price LH, 246, 341

Primus RJ, 307

Q

Quitkin FM, 113

\section{$\mathbf{R}$}

Ravert HT, 175

Redwine L, 100

Rezai K, 230

Rhodes JL, 325

Rimodini R, 82

Rinker CM, 57

Rintelmann J, 241

Rivier JE, 34

Robinson TE, 130

Roth $\mathrm{RH}, 92$

Rotondo A, 18

S

Salzmann JK, 175

Satoh K, 27

Schilström B, 44

Schmidt M, 391

Schöllnhammer G, 246

Secunda S, 110
Seibyl JP, 162

Serby M, 264

Sershen $\mathrm{H}, 202$

Sheitman BB, 205

Shiah I-S, 258, 382

Siever LJ, 264

Silverman J, 264

Slate SO, 360

Somes C, 34

Steinberg BJ, 264

Stewart JW, 113

Stokes P, 110

Sundstrom I, 370

Suzuki Y, 279

Svensson TH, 44, 151, 197

Swann A, 110

Szymanski S, 175

\section{T}

Tam E, 258

Tang C, 402

Tani K, 274, 279

Tarazi FI, 186

Taylor BP, 113

Tham C-S, 197

Tran A, 92

Trestman R, 264

Trujillo KA, 353

Tsukamoto T, 274, 279

\section{U}

Unlap MT, 12

V

van der Velde EA, 284 van Kempen GMJ, 284

van Louden $L, 284$

Vansell NR, 67

\section{W}

Wang Z-C, 279

Watkins GK, 230

Weston S, 264

Widmark C, 402

Wiegant VM, 284

Wilson PD, 175

Wong DF, 175

Wu JC, 402

\section{X}

Xu D-S, 279

Xu H-D, 279

Y

Yatham LN, 258, 382

Yeghiayan SK, 186

Yehuda R, 100

Yevich E, 307

Yokoi F, 175

Yoshikawa T, 100

Young EA, 353

Youngren KD, 92

Yuwiler A, 350

\section{Z}

Zametkin AJ, 391

Zis AP, 258, 382

Zoghbi SS, 162 